\title{
DISTRIBUCION Y ESTRUCTURA DE LA POBLACION DE ABIES RELIGIOSA (H.B.K.) SCHL. ET CHAM., EN EL COFRE DE PEROTE, ESTADO DE VERACRUZ, MEXICO'
}

\author{
Lazaro Rafael Sanchez-Velasquez \\ Ma. del Rosario Pineda-Lopez \\ Laboratorio Natural Las Joyas \\ de la Sierra de Manantlán \\ Universidad de Guadalajara \\ Niños Héroes 53 \\ El Grullo, Jal. \\ C.P. 48740
}

e
Instituto Nacional de Investigaciones sobre Recursos Bióticos ${ }^{2}$
$\mathrm{Km} 3$ carretera vieja a Coatepec
Xalapa, Ver.

\author{
Amador Hernandez-Martinez \\ Instituto Nacional de Investigaciones \\ sobre Recursos Bíticos ${ }^{2}$ \\ $\mathrm{Km} 3$ carretera vieja a Coatepec \\ Xalapa, Ver.
}

\begin{abstract}
RESUMEN
Se usó la frecuencia relativa y las correlaciones múltiples paso a paso para determinar las condiciones de altitud, pendiente y exposición bajo las cuales habita Abies religiosa (H.B.K.) Schl. et Cham. en la región del Cofre de Perote, estado de Veracruz, México.

El estudio también incluyó el análisis de la estructura de edades de la población, así como la relación entre el Incremento Corriente Anual (ICA), las variables arriba mencionadas y los atributos tales como cobertura, área basal del arbolado en pie y área basal de tocones.
\end{abstract}

\footnotetext{
1 Este trabajo formó parte del proyecto Cofre de Perote cuyo financiamiento fue otorgado por el Consejo Nacional de Ciencia y Tecnología a través del Programa Conservación Ecológica del Instituto Nacional de Investigaciones sobre Recursos Bióticos.

2 Institución desaparecida por decreto presidencial en 1988.
} 
Los resultados indican que los bosques de oyamel se encuentran en condiciones de pendiente y altitud restringidas, hecho que se atribuye principalmente al reemplazo de la masa forestal por cultivos agrícolas. En la zona de estudio, la pendiente y la altitud que resultaron con mayor probabilidad (0.579) de encontrar a Abies religiosa correspondieron a los intervalos de 40-55\% y de 3200-3400 metros, respectivamente. La exposición NW fue la más frecuente $(58 \%)$ entre los sitios de oyamel muestreados.

Se obtuvo una alta relación $(r=-0.96)$ entre el diámetro normal (DN) de los troncos y la edad. Asimismo, se estimó la estructura de edades que resultó ser característica de una especie tolerante a la sombra. Finalmente se encontró una relación positiva entre el ICA con respecto al área basal de los tocones y la pendiente $(r=0.77, p<0.001)$.

\section{ABSTRACT}

Analysis of relative frequency and multiple correlations were used to determine altitude, slope and exposure of places where Abies religiosa (H.B.K.) Schl. et Cham. is found in the Cofre de Perote area, state of Veracruz, México.

This study includes a population structure analysis, as well as an analysis of the relationships between the common annual increment (CAI), the variables mentioned above and the coverage, the standing tree basal areas, and the stump basal areas.

The results show that the fir forest is restricted to certain slope and altitude conditions. This is due primarily to the loss of forested land to agriculture. There is a 0.579 probability of finding fir trees at altitudes between 3200 and 3400 meters and on slopes of $40 \%$ to $55 \%$. With respect to exposure, the NW side shows the greatest frequency $(58 \%)$.

A high correlation $(r=-0.96)$ was found between the normal diameter (ND) and the age of the trees. Moreover, the age structure is that of a shade-tolerant species. Finally, a positive relationship was found between $\mathrm{CAl}$, the stump basal area and the slope $(r=0.77, p<0.001)$.

\section{INTRODUCCION}

El conocimiento de la influencia que los diferentes gradientes ambientales ejercen sobre los atributos de las especies vegetales es de gran significancia para su conservación y manejo, especialmente para aquellas que se encuentran bajo condiciones restringidas y amenazadas o en peligro de extinción como resultado de las actividades antrópicas. Lo anterior es importante debido a que cada especie responde de diferente manera a las condiciones ambientales (Billings, 1974). Por otro lado es bien sabido que los atributos poblacionales nos sirven de base para entender los patrones de la dinámica de las especies (Harper, 1977; Silvertown, 1982).

Entre los árboles de interés forestal, las coniferas son de mayor trascendencia económica actual en nuestro país y en el resto de Norteamérica. Dentro de este grupo se encuentra el género Abies, y en particular Abies religiosa (H.B.K.) Schl. et Cham. es una de las especies que por las propiedades físicas y mecánicas de su madera ha sido muy apreciada en los lugares donde se encuentra (Madrigal, 1967). Por ello es de suma importancia conocer los factores asociados a los bosques de oyamel y su distribución para generar estrategias dirigidas a su manejo sostenido y conservación in situ y ex situ.

El bosque de Abies en el Cofre de Perote suma un total de 1145 ha distribuidas en forma discontinua (Hernández-Martínez, 1984), esta superficie se va reduciendo a pequeños manchones debido a presiones antrópicas (talas, cultivos y actividades pecuarias). 
Los bosques del Cofre de Perote, incluyendo los de oyamel, han sido explotados clandestinamente desde hace mucho tiempo a pesar de la declaratoria de veda en este Parque Nacional (Vargas-Márquez, 1984). Además del mal uso de sus recursos bióticos, esta region se ha convertido en la actualidad en una fuente potencial de conflictos sociales (Golberg, 1983).

El Cofre de Perote o Nauhcampantépetl es un macizo montañoso que se encuentra situado sobre el paralelo $19^{\circ} 3^{\prime} \mathrm{N}$ y el meridiano $97^{\circ} 10^{\prime} \mathrm{W}$, cerca del límite entre los estados de Veracruz y Puebla. Forma parte del Eje Neovolcánico Transversal. El área de estudio abarca una parte de los municipios de Perote, Xico y Ayahualulco (Hernández-Martínez, 1984).

La estación metereológica que proporciona datos más confiables y más cercana a la zona de estudio, es la de Tembladeras, municipio de Xico, Ver. Según la clasificación de Köppen modificada por García (1981), el clima corresponde al tipo semifrío húmedo, con verano fresco corto. La temperatura y precipitación media anual son $9.1^{\circ} \mathrm{C}$ y 1577.5 $\mathrm{mm}$, respectivamente (Hernández-Martínez, 1984).

El presente trabajo tiene como objetivo determinar algunos de los factores abióticos asociados al bosque de $A$. religiosa, así como la distribución de edades de la población de esta especie dominante.

\section{METODOLOGIA}

Mediante fotointerpretación se elaboró un plano para delimitar la zona de bosques de oyamel. Las fotografías aéreas que se utilizaron fueron tomadas en agosto de 1982. Se eligieron aleatoriamente 19 sitios de muestreo, trazando en cada uno círculos concéntricos de 80,400 y $1000 \mathrm{~m}^{2}$. En el primero se midieron todos los renuevos de Abies con diámetro normal (DN) (diámetro a la altura de $1.3 \mathrm{~m}$ de la base del árbol) menor o igual a $4.9 \mathrm{~cm}$, en el segundo todos los árboles de 5 a $14.9 \mathrm{~cm}$ de DN, y en el tercero todo el arbolado mayor de $15 \mathrm{~cm}$ de DN. También se registró la altura y el diámetro de los tocones, la profundidad de la capa de musgos y de la hojarasca, la altitud, la exposición y el porcentaje de cobertura del estrato arbóreo. Esta última variable se midió con la ayuda de un clinómetro sostenido en posición vertical, se anotó la presencia o ausencia del dosel superior en trece puntos elegidos aleatoriamente dentro de cada uno de los sitios. Durante el trabajo de campo se tomaron muestras de anillos de crecimiento a 107 árboles de diferentes clases diamétricas con un taladro de Pressler, con la finalidad de conocer la relación entre el diámetro de los troncos y la edad de la población.

La relación entre la altitud y la exposición y así como entre la altitud y la pendiente se obtuvo con base en la frecuencia relativa, es decir, se consideró la proporción del número de veces en el que los sitios aparecieron en cada una de las clases combinadas de tamaño dentro de estas variables. Por ejemplo, de 19 sitios muestreados $47.4 \%$ se localizan con la exposición de 270 a 360 grados y a una altitud de 3200 y $3400 \mathrm{~m}$.

La relación entre la edad $(X)$ y el DN $(Y)$ se determino con ayuda de la simulación de varios modelos lineales (elaborados con transformaciones de $X$ y $Y$ ). Se seleccionó aquel que presentó el valor máximo de correlación, el mínimo de la suma de cuadrados mínimos del error del ANVA (análisis de varianza) y el que mejor reprodujo una tendencia 
parecida al comportamiento de los datos observados. Se procedió de la misma manera para elegir la función que mejor representara la distribución de edades de la población (edad $(X)$ vs. número de individuos $(Y)$ ).

La correlación entre el Incremento Corriente Anual (ICA) y los factores abióticos se determinó paso a paso para detectar las variables que mejor explicaran la variación del incremento diamétrico de $A$. religiosa. Del mismo modo se hizo con los atributos de la éspecie (área basal de tocones, área basal en pie y cobertura), combinando ambos tipos de variables.

Para extrapolar los diámetros de los tocones (que tuvieron alturas menores de $1 \mathrm{~m}$ ) a valores correspondientes a $1.3 \mathrm{~m}$ de altura (DN), se realizaron mediciones de 152 individuos en dos diferentes alturas: $0.3 \mathrm{~m}$ y $1.3 \mathrm{~m}$. Utilizando una regresión lineal simple se estimó el DN de los tocones y posteriormente el área basal de los árboles que se habían extraido en cada sitio.

\section{RESULTADOS Y DISCUSION}

Relación entre el área basal en pie y la altitud

En la figura 1 se observa una falta de asociación entre el área basal en pie por hectárea y la altitud: Abies religiosa casi no se presenta por encima de los 3600 y tampoco abajo de los $2800 \mathrm{~m}$, sus valores máximos están concentrados en el intervalo de 3200 a $3400 \mathrm{~m}$ de altitud llegando a alcanzar más de $70 \mathrm{~m}^{2} \mathrm{ha}^{-1}$. Los factores limitantes de las poblaciones de $A$. religiosa arriba de los $3600 \mathrm{~m}$ s.n.m. son sin duda las condiciones de menor humedad que prevalecen en México más allá de esta altitud, y que no parecen ser favorables para la existencia de especies del género Abies (Rzedowski, 1981). Asimismo, la especie no se encuentra por abajo de los $2800 \mathrm{~m}$ probablemente porque en tales altitudes Pinus tiene mayor éxito competitivo que Abies.

Relación entre la frecuencia del bosque de oyamel, la altitud y la pendiente

La figura 2a presenta el cuadro probabilístico de la ubicación del bosque de oyamel en función de la altitud y de la pendiente. La comunidad se encontró en pendientes que van de 20 a $65 \%$, situación muy similar a la observada en el Valle de México: 17 a $60 \%$ (Madrigal, 1967).

De la Fig. 2 a se deduce que la mayor probabilidad de existencia del bosque (58\%) se localiza entre 40 y $50 \%$ de pendiente y entre 3200 y 3400 m de altitud, indicando que los bosques de oyamel se presentan mayormente en la región en declives pronunciados. Tal situación se le atribuye a las presiones antropicas existentes en la zona de estudio, que han provocado que en las laderas menos inclinadas la masa forestal resulte sustituida por cultivos de papa y haba, entre otros.

Comparativamente, en el Valle de México el bosque de Abies tampoco se observa en lugares planos o poco inclinados, pero tal hecho quizá es consecuencia también de la escasez de estos terrenos en la zona montañosa y, en parte, de la influencia humana (Rzedowski, 1981). 


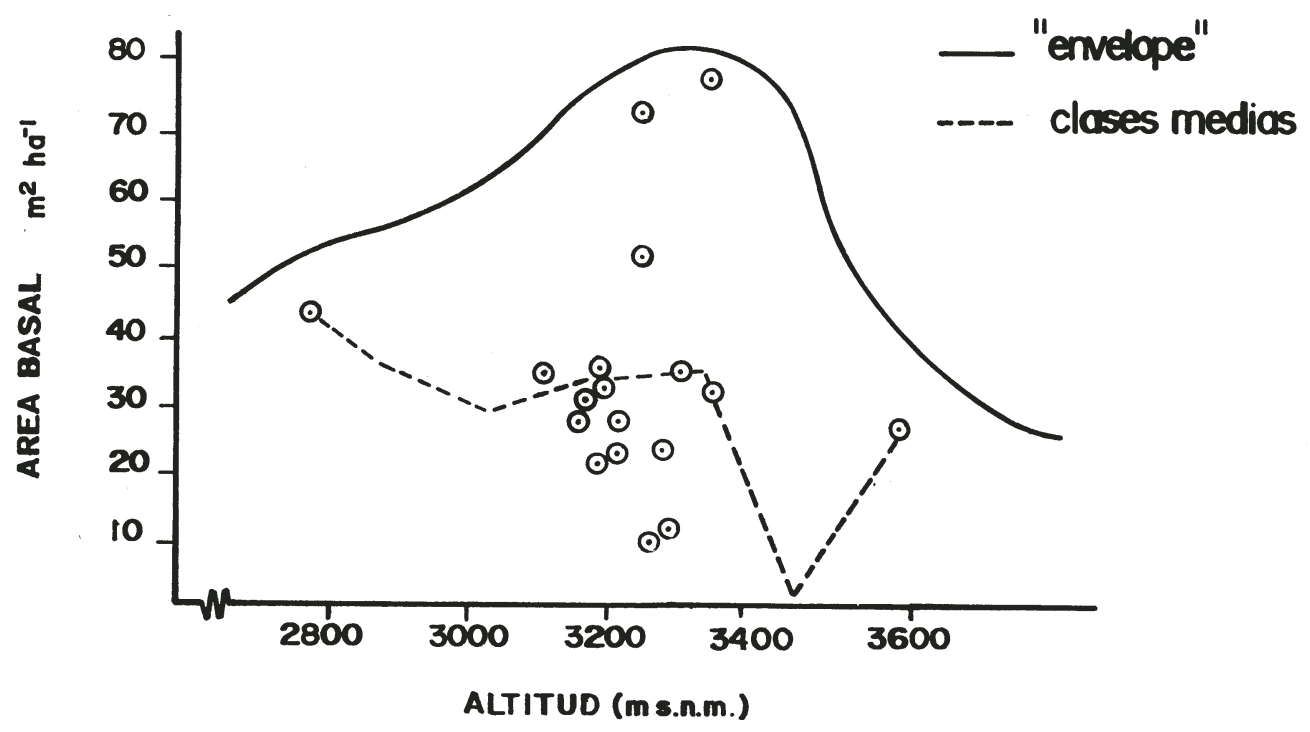

Fig. 1. Relación entre el área basal en ha ${ }^{-1}$ de Abies religiosa y la altitud en el Cofre de Perote, estado de Veracruz, México.

Relación entre la frecuencia del bosque de oyamel, la exposición y la altitud

La figura $2 b$, muestra la frecuencia relativa de Abies religiosa en relación con estas dos variables, señalando que $47 \%$ de los sitios tienen exposición NW, a una altitud entre 3200 y $3400 \mathrm{~m}$.

Para explicar este último hecho no contamos con la información suficiente en el aspecto fisiológico ni ecológico de la especie. Sin embargo, es probable que en esta exposición se den las condiciones ambientales propicias para que los individuos de $A$. religiosa sean competitivamente superiores a los de otras especies. Con base en observaciones realizadas en lugares donde frecuentemente se encuentran los oyameles, Chapa (1976) considera como un requerimiento la exposición $N$ y E para el establecimiento de plantaciones de estos árboles.

En general, en el Cofre de Perote se encontró A. religiosa en las siguientes exposiciones; SW, W, NW, N y NE (Fig. 2b). A diferencia de lo registrado por Madrigal (1967) 


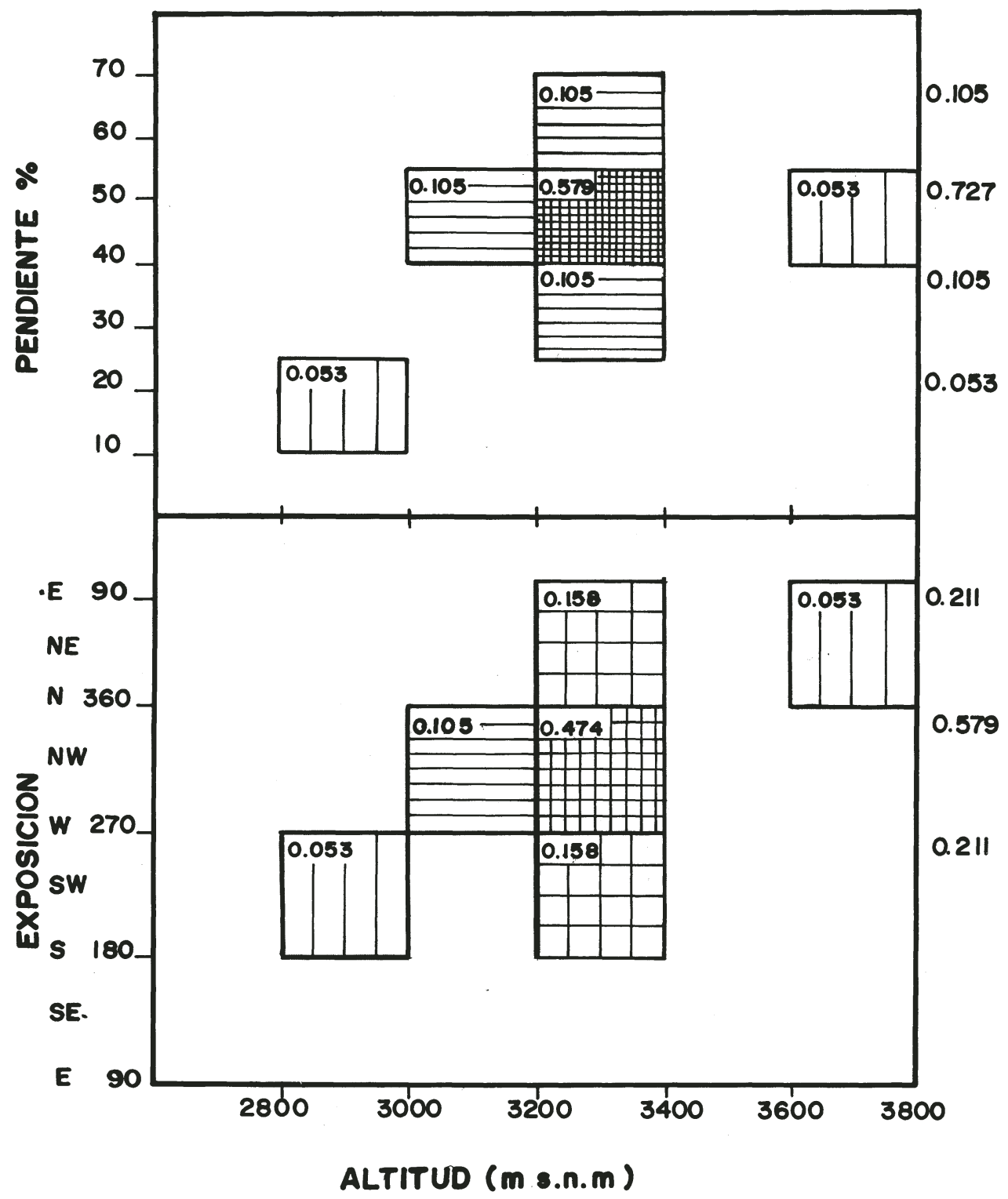

Fig. 2. Modelo predictivo para la probabilidad de existencia de Abies religiosa en "celdillas" combinadas con altitud vs. pendiente a) y exposición b). En el margen derecho se señala la suma de probabilidades para cada intervalo señalado en el margen izquierdo. 
para el Valle de México, dicha especie no se encontró en la exposición S y SE, y sí en la NW y NE.

Relación edad-diámetro normal (DN) y la estructura de edades

El modelo lineal que mejor explica la relación edad-DN (se eligió aquel que presentó el valor máximo de correlación, el mínimo de la suma de cuadrados mínimos del error en el ANVA y el que mejor representara el comportamiento de los datos observados) resulto ser la función:

$$
Y=\frac{X}{0.00357 X+1.3887}
$$

donde $X$ es la edad y $Y$ el $D N(P<0.001)$ (véase la Fig. 3).

La misma gráfica ilustra también la estructura de edades de la población de Abies religiosa. El ajuste fue significativo al nivel de 0.05 . La función resultante fue:

$$
Y=\frac{1}{11.3-\ln X(4.8)}
$$

donde, $Y$ es el número de individuos y $X$ la edad. El valor de correlación es $r=-0.96$. La forma de la curva (Fig. 3) nos muestra una población típica de una especie tolerante a la sombra (Martínez-Ramos, 1985), la cual esta regenerándose bajo su propio dosel. Esta curva indica que existe un número alto de individuos de edades jóvenes y establecidos, lo que señala que el bosque puede tener una buena regeneración, establecimiento y reclutamiento entre las diferentes clases de tamaño. Asimismo, cabe interpretar que cuando la población va madurando, el número de individuos de cada cohorte disminuye, debido a que comienza a operar la competencia intra e interespecifica (por espacio y nutrimentos) provocando lo que se denomina autoaclareo (ver Jardel y Sánchez-Velásquez, 1989). A través del tiempo relativamente pocos individuos llegan a la edad adulta y contribuyen a la reproducción. Esto nos sugiere que si tomamos el valor de $Y$ (número de individuos) $y$ el de $X$ (edad) como la proporción de sobrevivientes y tiempo, respectivamente, obtendremos para la población de Abies una curva de sobrevivencia de tipo III de Deevey (1947). Lo anterior va de acuerdo con la aplicabilidad de tablas de vida estáticas para poblaciones de individuos longevos (Harper, 1977; Silvertown, 1982).

Area basal de tocones

De la medición de los diámetros de los troncos de 152 individuos a dos diferentes alturas $(0.3$ y $1.3 \mathrm{~m}(\mathrm{DN}))$, se encontró una relación altamente significativa $\left(P_{\leq} 0.001\right.$, $r=0.99$ ) entre el DN y el diámetro de la base. La función ajustada fue: $Y=0.87 X+0.3041$, donde $Y$ es el diámetro de la base y $X$ el DN. Con fundamento en esta ecuación se calculó el área basal a $1.3 \mathrm{~m}$ de altura (DN) de los árboles extraidos (tocones) para cada uno de los sitios muestreados, lo que a su vez se empleo para analizar su influencia, junto con otros factores, sobre el Incremento Corriente Anual (ICA). 


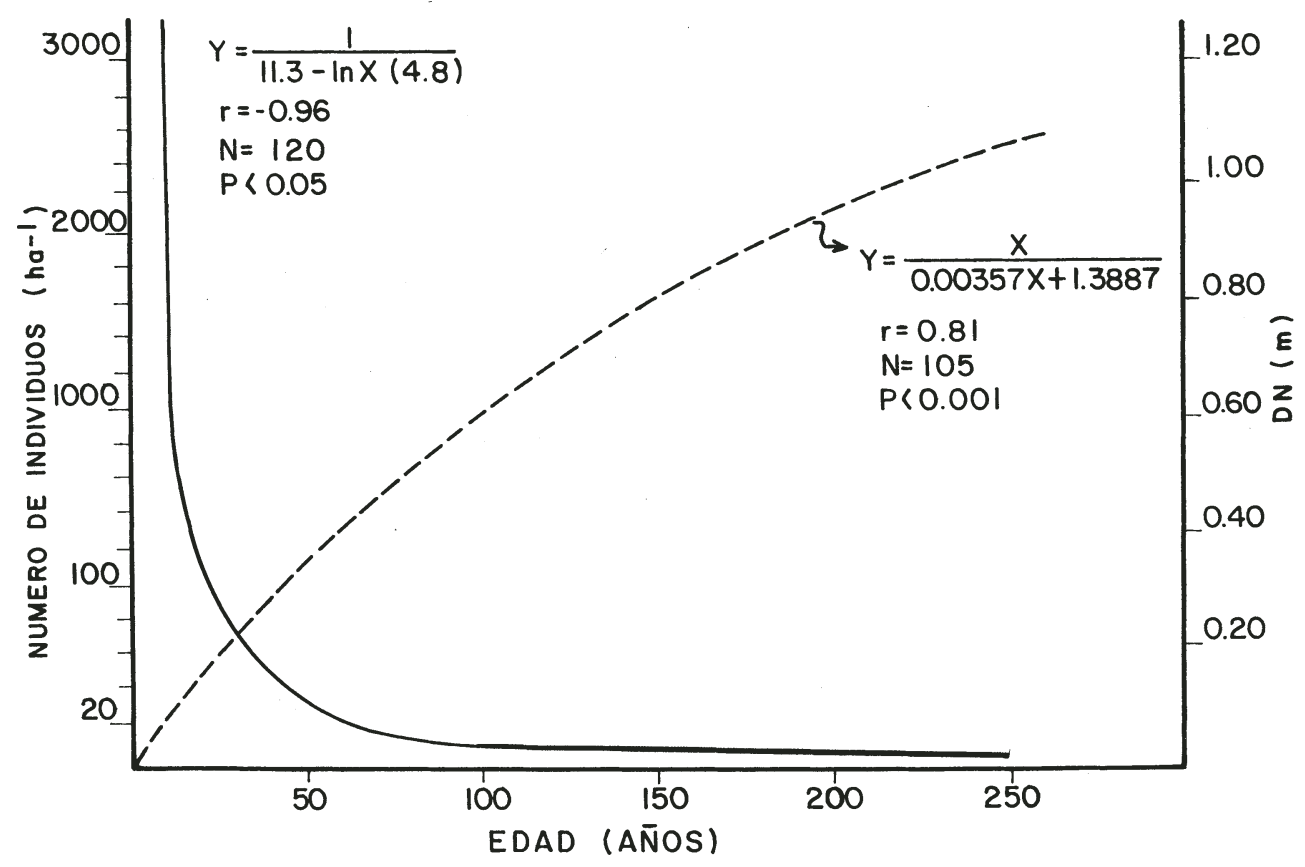

Fig. 3. Gráfica que ilustra la relación positiva entre la edad y el diámetro normal (DN). Muestra asimismo la estructura de edades de la población de Abies religiosa en el Cofre de Perote, estado de Veracruz, México.

Correlaciones entre el ICA y algunos factores ambientales

Con base en las muestras obtenidas con el taladro de Pressler, el ICA fue determinado en un tctal de 107 árboles de oyamel, calculando el ensanchamiento promedio de los últimos 10 anillos de crecimiento. Posteriormente se analizó el ICA en relación a la cobertura, área basal de los tocones y área basal del arbolado en pie (variables intrínsecas), utilizando correlaciones múltiples paso a paso (Cuadro 1). Estos tres factores tienen una correlación con el ICA de $r=0.63 \quad(p=0.92)$, de ellos el que mejor explica la variación del ICA es el área basal de los tocones $(r=0.61, p=0.004)$, lo cual señala que el ICA responde positivamente en aproximadamente $40 \%$ a la extracción. Lo anterior se 
Cuadro 1. Correlaciones entre el ICA de Abies religiosa (H.B.K.) Schl. et Cham. y algunos factores ambientales en el Cofre de Perote, estado de Veracruz, México.

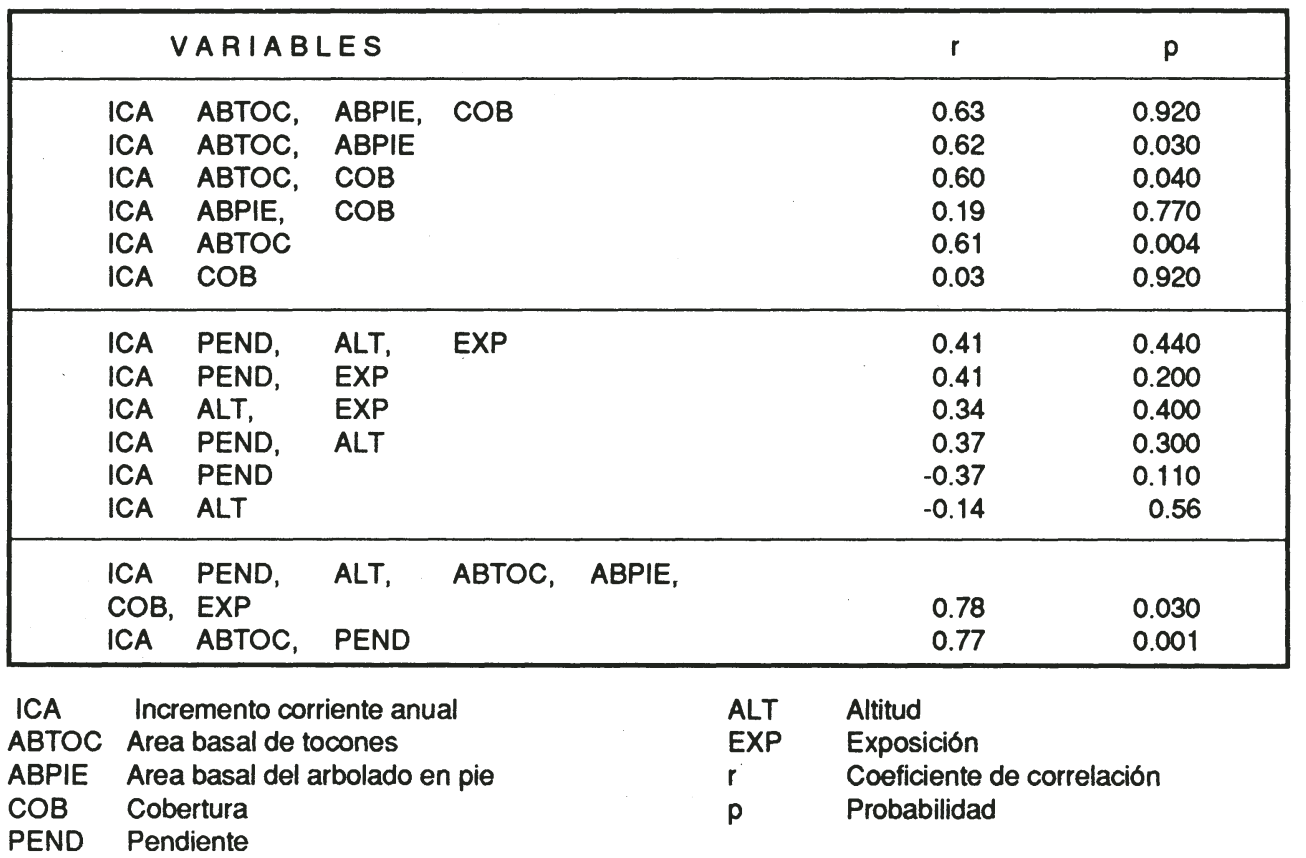

infiere en función del hecho de que el ICA se estimo con base en los últimos 10 anillos de crecimiento y por lo tanto quedó sujeto al efecto de la liberación en el arbolado residual.

El análisis de la correlación múltiple paso a paso entre el ICA frente a la pendiente, la exposición y la altitud (variables extrínsicas) (Cuadro 1), muestra que no existe una vinculación significativa entre estos parámetros $(r=0.41, p=0.44)$, siendo la pendiente la que mayor influencia mostró.

Entre el ICA y las variables intrínsecas y extrínsecas (Cuadro 1), se encontro una alta correlación $(r=0.78, p=0.03$ ). No obstante, al analizar el conjunto de las variables con mayor correlación de cada grupo (área basal de tocones y pendiente), éstas resultaron ser las que más influyen sobre la variabilidad del ICA $(r=0.77, p=0.001)$. La pendiente como factor aislado no tiene gran significancia en relación con el ICA, situación muy similar a lo concluido por Manzanilla (1971) para A. religiosa en el Valle de México. Es claro que dado que éstas son las variables que más influencia tienen sobre el ICA, no se pueden definir óptimos de fácil manipuleo que logren mayores incrementos, razón por la cual no se concretaron las regresiones múltiples. Sin embargo, la correlación positiva entre el ICA y el área basal de los tocones muestra que A. religiosa responde muy bien a los aclareos, - sea que al eliminar individuos de su misma especie, los restantes responden bien a la disminución de la competencia intraespecífica, observándose mayores incrementos en 
el ICA. Lo que se esperaba era encontrar una muy buena relación entre el ICA y el área basal en pie para emanar de ahl recomendaciones de manejo. En realidad, esta relación resultó ser mínima (Cuadro 1), debido seguramente a las intensidades desiguales de corta, y en general a las diferencias en la historia de los sitios.

Cabe enfatizar que la comprension de las relaciones entre $A$. religiosa y los factores abióticos (altitud, pendiente y exposición) se ve distorcionada por la actividad humana, que restringe a esta especie a lugares no aptos para la agricultura.

Por otro lado, a diferencia de lo registrado por otros (Crow y Weaver, 1977; Ogden, 1978, 1985; Palmer y Ogden, 1983; Knowles y Grant, 1983; Weaver, 1989), se encontró una alta correlación entre la edad y el diámetro de los troncos $(r=0.81, P<0.001)$, razón por la cual pudimos estimar mediante una regresión lineal simple la distribución de edades de la población de $A$. religiosa (Fig. 3). Esta distribución nos indica que, al haber un alto número de individuos juveniles, cuyo número va disminuyendo gradualmente a través de las clases de tamaño más grandes, la población tiene un buen reclutamiento para cada clase de tamaño. Con lo anterior se infiere que la población de Abies religiosa se ha estado regenerando en forma activa y sostenida.

\section{CONCLUSIONES}

El bosque de oyamel existe con mayor frecuencia en sitios con pendientes que oscilan entre 40 y $55 \%$ y en altitudes entre 3200 y $3400 \mathrm{~m}$. Esto último es resultado de las presiones antrópicas, pues los cultivos agrícolas desplazan al bosque en las partes menos inclinadas. La exposición NW fue la más frecuente (58\%) entre los sitios de oyamel muestreados.

$$
\text { La ecuación } Y=\frac{X}{0.00357 X+1.3887} \quad \text { resultó ser la que mejor se ajustó }
$$

para explicar la relación entre la edad $(X)$ y el diámetro normal $(Y)(r=0.81, p=0.001)$. La estructura de edades de la población de oyamel estimada con la ayuda de esta relación resultó ser típica de una especie tolerante a la sombra que se regenera abajo de un dosel cerrado (Martínez-Ramos, 1985).

De los factores analizados en relación con el ICA (Incremento Corriente Anual), el área basal de los tocones y la pendiente son los que mejor explicaron su variación $(r=0.77$, $p=0.001$ ).

\section{AGRADECIMIENTOS}

Los autores agradecen a la M. en C. Laura Snook (Directora del Proyecto Cofre de Perote), por el apoyo y las facilidades otorgadas para la realización de esta investigación, y al Dr. Charles Peters por sus valiosas y atinadas sugerencias en el proceso de análisis de este trabajo. Asimimo, agradecemos al M. en C. Manuel Pío Rosales A. y a tres revisores anónimos por sus valiosos comentarios, los cuales hicieron que mejorara sustancialmente la versión final del mismo. 
Sánchez Velásquez et al.: Distribución y Estructura de Abies religiosa en el Cofre de Perote

\section{LITERATURA CITADA}

Billings, W. D. 1974. Environmental: concept and reality. In: Strain, B .R. y W. D. Billings (eds.). Vegetation and environment. Handbook of vegetation science. Part. VI. Junk. The Hague. pp 9-35.

Crow, T. R. y P. L. Weaver. 1977. Tree growth in a moist tropical forest of Puerto Rico. Institute of Tropical Forestry. Forest Service. U. S. Department of Agriculture. Res. Pap. ITF-22. Rio Piedras, Puerto Rico. $17 \mathrm{pp}$.

Chapa B., M. del C.1976. Principales técnicas de cultivo para árboles de navidad. Instituto Nacional de Investigaciones Forestales. Bol. de Div. No. 41. 85 pp.

Deevey, E. S. 1947. Life tables for natural population of animals. Quart. Rev. Biol. 22: 283-314.

Flores-Mata, G., Jiménez-López, J., Madrigal-Sánchez, X., Moncayo-Ruiz, F. y F. Takaki-Takaki. 1971. Memoria del mapa de tipos de vegetación de la República Mexicana. Secretaría de Recursos Hidráulicos. México, D.F. 59 pp.

García, E. 1981. Modificación al sistema de clasificación climática de Köppen. Instituto de Geografia. Universidad Nacional Autónoma de México, México, D.F. 252 pp.

Golberg, A. D. 1983. El Cofre de Perote: investigaciones ecológicas en un área conflictiva. Instituto Nacional de Investigaciones sobre Recursos Bióticos. Cuadernos de Divulgación. No. 9. 63 pp.

Harper, J.L. 1977. Population biology of plants. Academic Press. London and New York. 892 pp.

Hernández-Martínez, A. 1984. Estructura y regeneración del bosque de oyamel (Abies religiosa Schl. et Cham.), en el Cofre de Perote. Tesis de Ingeniero Agrónomo Especialista en Bosques. Universidad Autónoma Agraria "Antonio Narro". Saltillo, Coahuila. 125 pp.

Jardel P., E. y L. R. Sánchez-Velásquez. 1989. La sucesión forestal: fundamento ecológico de la silvicultura. Ciencia y Desarrollo 14(84): 33-43.

Knowles, P. y M. C. Grant. 1983. Age and size structure analysis of Engelmann spruce, ponderosa pine, lodgepole pine, and limber pine in Colorado. Ecology 64(1): 1-9.

Madrigal S., X. 1967. Contribución al conocimiento de la ecología de los bosques de oyamel (Abies religiosa (HBK.) Schl. et Cham.) en el Valle de México. Instituto Nacional de Investigaciones Forestales. Bol. Téc. no. 18. 95 pp.

Manzanilla, H. 1971. Influencia de algunos factores silvicolas sobre el ICA de Abies religiosa. Instituto Nacional de Investigaciones Forestales. Bol. Téc. no. 38.45 pp.

Manzanilla, H. 1974. Investigaciones epidométricas y silvicolas en bosques mexicanos de Abies religiosa. Secretaría de Agricultura y Ganadería. México, D. F. 165 pp.

Martínez-Ramos, M. 1985. Claros, ciclos vitales de los árboles tropicales y regeneración natural de las selvas altas perennifolias. In: Gómez-Pompa, A. y S. Del Amo R. (eds.). Investigaciones sobre la regeneración de selvas altas en Veracruz, México. Vol. 2. Instituto Nacional de Investigaciones sobre Recursos Bióticos. Alhambra Mexicana. México, D. F. pp. 191-239.

Miranda, F. y E. Hernández X., 1963. Los tipos de vegetación de México y su clasificación. Bol. Soc. Bot. Méx. 28: 29-179.

Ogden, J. 1978. On the diameter growth rates of red beech (Notofagus fusca) in different parts of New Zealand. New Zealand Journal of Ecology 1: 16-18.

Ogden, J. 1985. An introduction to plant demography whith special reference to New Zealand trees. New Zealand Journal of Botany 23: 751-772.

Palmer, J. y J. Ogden. 1983. A dendrometer in kauri (Agathis australis). New Zealand Journal of Botany 2: $121-126$.

Rzedowski, J. 1981. Vegetación de México. Limusa. México, D.F. 431 pp.

Rzedowski, J. y R. McVaugh. 1966. La vegetación de Nueva Galicia. Contrib. Univ. Mich. Herb. 9(1): $1-123$.

Silvertown, J. W. 1982. Introduction to plant population ecology. Longman. London. 209 pp.

Vargas-Márquez, F. 1984. Parques nacionales de México y reservas equivalentes. Instituto de Investigaciones Económicas. Universidad Nacional Autónoma de México. México, D.F. 226 pp.

Weaver, P. L. 1987. Ecological observations on Magnolia splendens Urban in the Luquillo Mountains of Puerto Rico. Carib. Jour. Sci. 23(3-4): 340-351. 